\title{
Calibration-less Anthropometric Scanner Using GPU's
}

\author{
Mario A. GAZZIRO ${ }^{\mathrm{a}, \mathrm{b}}$, Pedro SCOTTON ${ }^{\mathrm{a}}$, Heitor BITTENCOURT ${ }^{\mathrm{b}}$, Andre OSTI ${ }^{\mathrm{a}}$ \\ ${ }^{a}$ ICMC - Universidade de Sao Paulo, Brazil; \\ IFSC - Universidade de Sao Paulo, Brazil
}

\begin{abstract}
Excess body fat, or obesity often cause significant harm to health. The development of a mechanism for evaluation of body fat more dynamic expedite the process of measurement. The goal of this work is the creation of a clinical analysis equipment capable of determining the percentage of fat in patients so rapidly, accurately, without the need for calibration after installation. A mechanical system has been developed by us in order to achieve acquire data over the whole human body. We conducted case study of a single evaluation a result compatible with the visual inspection performed by experts.
\end{abstract}

Keywords: 3d body scanning, Body Mass Index, GPU,

\section{Introduction}

Excess body fat, or obesity often cause significant harm to health, and today considered a disease and according to the WHO (World Health Organization), which implies diminution of the quality and life expectancy.

The development of a mechanism for evaluation of body fat more dynamic expedite the process of measurement, making it possible to carry out assessments in a larger number of people in a given population in a short period of time, simplifying the task of determination of regional profiles of obesity. A greater precision equipment, namely, providing a more reliable, tends to encourage individual efforts of those who are in treatment.

In the scenario of gyms where are held most evaluations of body mass index are performed, it is rare to find a technician with the skills to perform calibration in $3 \mathrm{~d}$ scanners. Due to this fact the elaboration of a device that does not require calibration after your installation is of great importance. Another aspect is the speed of the test, which should be fast because the number of people assessed. To accomplish the procedures more quickly, the use of acceleration processing with general-purpose graphics cards (GPUs) was necessary.

\section{Objectives}

The goal of this work is the creation of a clinical analysis equipment capable of determining the percentage of fat in patients so rapidly, accurately, without the need for calibration after installation.

\section{Methodology}

We chose to use Microsoft(TM) Kinect(TM) sensor as applied to KinectFusion platform [1], also from Microsoft(TM) for the acquisition and registration of point clouds. To speed up the process, a GPU card from NVIDIA was used, model GTX680 with 1538 cuda cores with 4 TeraFLOPs of computational power (single precision).

In order to achieve acquire data over the whole human body, a mechanical system has been developed by us, shown in Figure 1 (CAD design) and Figure 2 (prototype). 


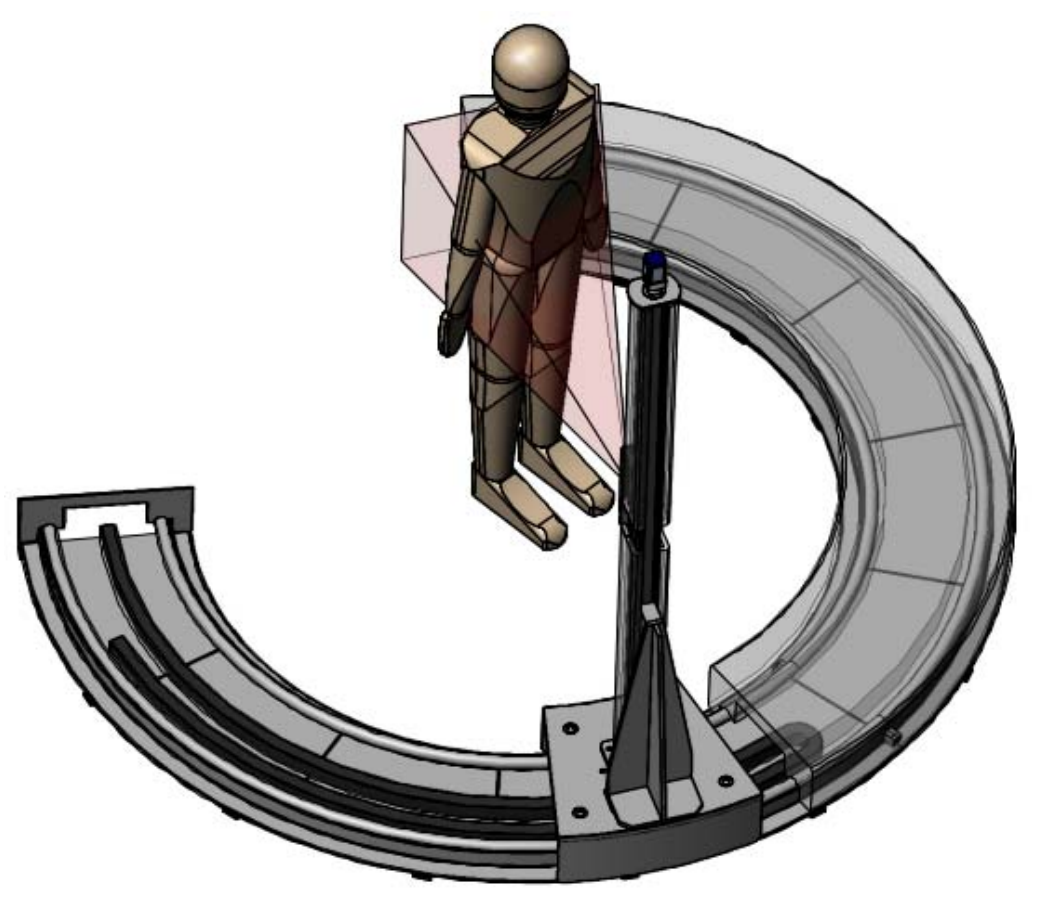

Fig. 1. Anthropometric Scanner CAD design.

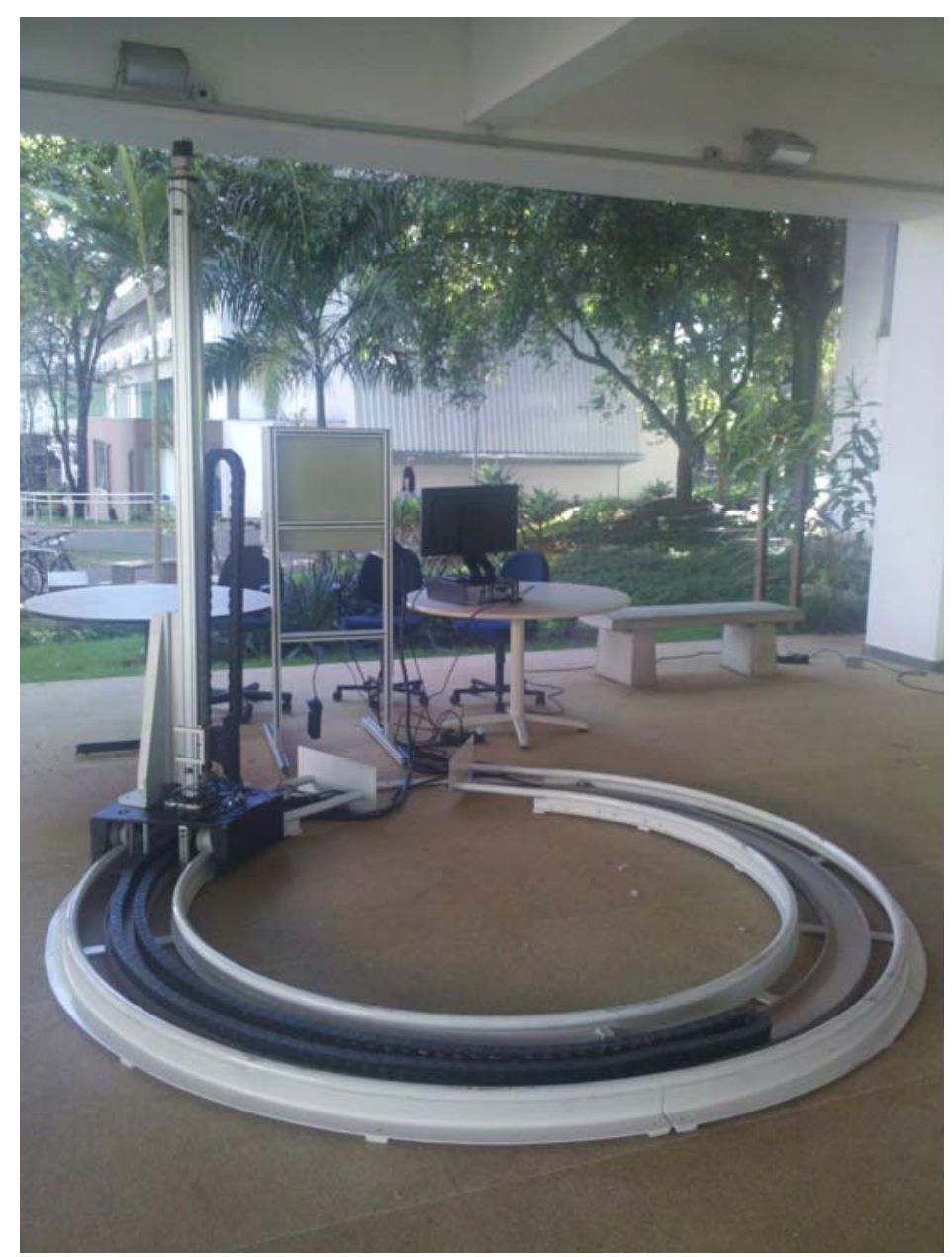

Fig. 2. Anthropometric Scanner Prototype. 
We need approximately 80 point clouds from different viewpoints to cover the area of the human body with the optical field of view of Microsoft(TM) Kinect(TM).

Once obtained and recorded the cloud points of the human body, the normal vectors are calculated for each face, and then computed the body volume (in $\mathrm{cm}^{3}$ ), using a technique based on Voronoi diagrams.

With the volume of the body, the next step is to weigh the body in a conventional balance. Having hands mass and volume to compute body density. Once obtained the body density, we use the equation of Siri [2] (equation 1) to achieve our goal of assessing body fat percentage (BF).

$$
B F=\left(\frac{4.95}{d}-4.50\right) * 100
$$

Another approach for obtaining an estimated weight is to conduct from the body surface area, eliminating the need for a scale in the system.

First we used the relation proposed by Yu et al [3] (equation 2), to estimate body surface area based on height and weight.

$$
S=0,015952 \sqrt{H * M}
$$

Then, based on Equations 1 and 2, we arrive at a formula (Equation 3) where the body fat percentage can be measured based on the volume of the body, height and area of supercifie, without the necessity of weighing the person.

$$
B F=\frac{495 * V * H}{1000\left(\frac{S}{0,015952}\right)^{2}}-450
$$

To get a good result in BF it is necessary to calculate with precision the volume of the person being measured. Since the point clouds generated by the Kinect contains much noise - even after the filtering platform KinectFusion - is necessary to perform a smoothing of the point cloud, using the method of Gois et al [4]. The results are presented in Figures 3 (a, b, c, d).

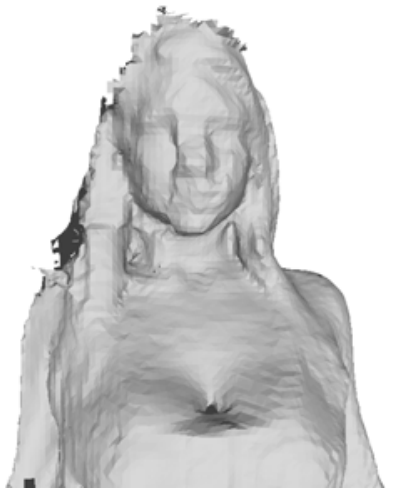

(a) Original surface

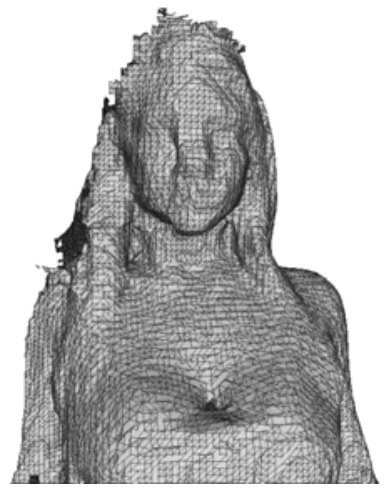

(c) Original mesh

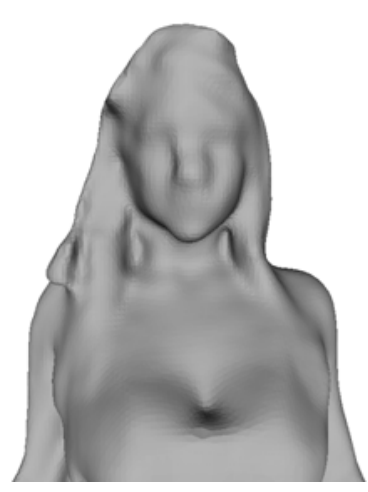

(b) Smoothed surface

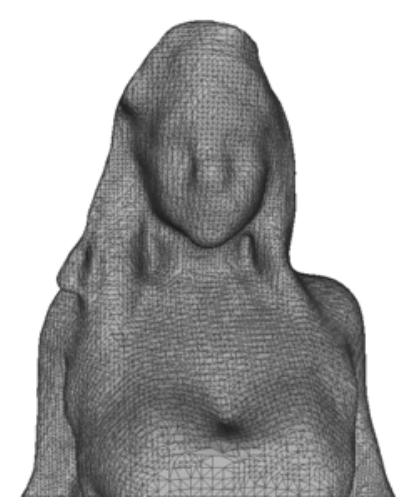

(d) Smoothed mesh

Fig. 3. Original and Smoothed surfaces and meshes from KinectFusion (a)(c) and after Gois [4] method (b)(d). 


\section{Results}

We conducted a single evaluation, with an individual female aged 23 years 49 kilos, height $1.68 \mathrm{~m}$ (Figure 4). The volume obtained was $60,110 \mathrm{~cm}^{3}$ and fat percentage estimated by the system was $22.3 \%$, a result compatible with the visual inspection performed by experts.
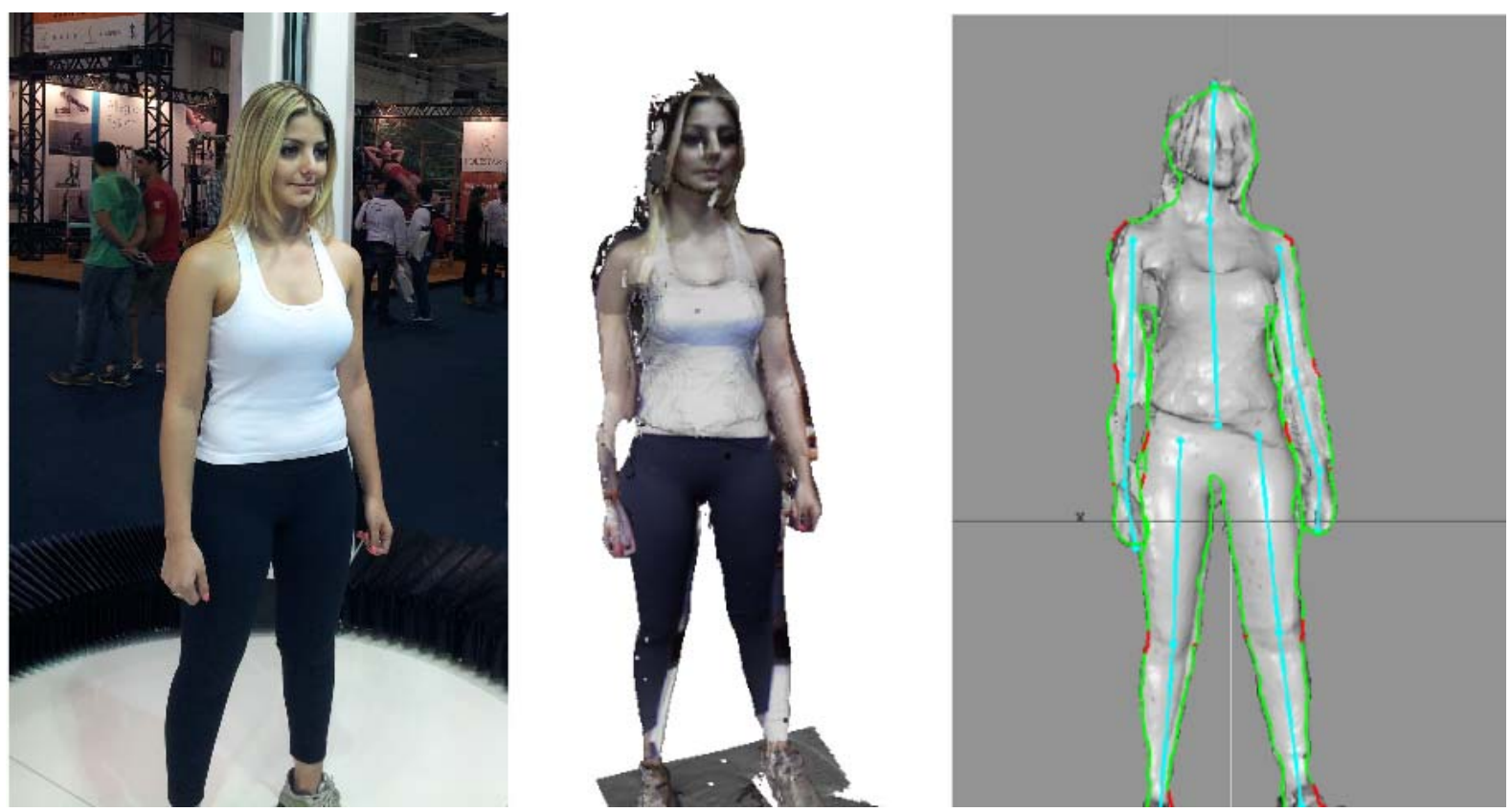

Fig. 4. Person being evaluated, triangular mesh with texture and point cloud with skeletonization and contour.

\section{Conclusion}

The results obtained in the evaluation of the fat percentage of patients evaluated are compatible with the visual inspection performed by experts. Even without the results of validating clinical - that will be done in further studies, with water tank and compare our results with the gold standard - the authors already consider satisfactory initial results, based on the success of the measures when compared to visual inspection.

\section{Acknowledgments}

The authors thanks the support of FAPESP (Research Council for the State of São Paulo) and researchers Soraia Musse, Jacques Junior Julio, John Paul Gois, Paulo Suzuki and our model Rebeca Giancursi.

\section{References}

1. Newcombe, R. A. et al. KinectFusion: Real-time dense surface mapping and tracking , ISMAR '11 Proceedings of the 2011 10th IEEE International Symposium on Mixed and Augmented Reality Pages 127-136,

2. Siri, W. E. (1956). The gross composition of the body, In: Advances in Biological and Medical Physics, pages 239-240. C. A. Tobias and J. H. Lawrence, New York.

3. Yu, C., Lo, Y., and Chiou, W. (2003). The 3d scanner for measuring body- surface area: a simplified calculation in the chinese adult. Applied Ergonomics, 34:273-278.

4. Gois, J. P. (2004). Reconstruçao de superficies a partir de nuvens de pontos. Master's thesis, Universidade de Sao Paulo, 120p.

5. Franca, J. G. D. M.; Gazziro, M. A,; Ide, A. N.; Saito, J. H. A 3D Scanning System Based on Laser Triangulation and Variable Field of View. In: Internation Conference on Image Processing, 2005, Gênova - Itália. Proceedings of the 12th International Conference on Image Processing (ICIP2005).. New Jersey - USA, 2005. v. 1. p. 425-428. 